\title{
Design of Underwater Robot Operation System Based on Visible Light Communication Technology
}

\author{
Xinqi $\mathrm{Li}^{1,2}$, Mengyu Yang ${ }^{1,2+}$, Lin $\mathrm{He}^{1,2}$ and Miao Sun ${ }^{1,2}$ \\ ${ }^{1}$ Tianjin University of Technology and Education, Tianjin, China \\ ${ }^{2}$ Tianjin Key Laboratory of Information Sensing \& Intelligent Control, Tianjin, China
}

\begin{abstract}
In this paper, visible light communication technology is applied to the operation system of underwater robot. The underwater robot in the system is controlled by STM32 single chip microcomputer. The blue LED light source of the underwater robot is used as the transmitting end of visible light communication, and the PIN silicon photodiode is used as the receiving end of visible light communication. The operation system network of underwater robot based on visible light communication technology is established, The functions of ranging, positioning, grasping and placing the target are realized.
\end{abstract}

Keywords: Visible light communication, Underwater robot, STM32, LED.

\section{Introduction}

\subsection{Visible Light Communication}

Visible light communication technology is a kind of wireless communication technology, which can transmit information by using the scintillation signal issued by the light-emitting diode. Compared with the traditional wired communication mode, it has the characteristics of high bandwidth, high rate, not susceptible to external magnetic field interference, and high flexibility[1-2]. At present, visible light communication technology has been applied in transportation, intelligent home, underwater work and other fields.

In the field of traffic, Okada et al. proposed to use a two-dimensional LED array as a transmitter and a two-dimensional image sensor as a receiver to form a parallel LED visible light communication system, and to use the spatial frequency component characteristics of the receiving pattern to set the distance of vehicles. In the field of intelligent home, Komine and others through the study of the layout, the design of unit of LED light, of visible light transmission channel (direct channel and the reflection channel), indoor personnel ambulate reflection caused by shadow, wall reflection, intersymbol interference will affect the performance of the system such as research, draw the different receiving field of view Angle and different data transfer rates under the influence of various factors on the system performance curve. In the field of underwater operation, Fangchen $\mathrm{Hu}[3]$ et al. compared the three modes of underwater acoustic communication, underwater radio frequency transmission and underwater visible light communication, and found that visible light communication has the advantages of high transmission rate and small loss of blue-green light in water when conducting underwater operation[4].

\subsection{Underwater Robot}

In recent years, underwater robot is an extreme operation robot, which has become an important tool and means of ocean development. This robot can replace people to operate underwater for a long time in highly dangerous environment, polluted environment and zero-visibility waters[5-7].

Gaochao Yan[8] et al. designed the simplified model framework of the underwater robot and carried out topology optimization on the framework, which reduced the quality of the underwater robot while reducing the deformation of the model and improved the comprehensive application ability of the underwater robot. Hao Zhang[9] et al. used load distribution control technology to design the energy storage device of the underwater robot. The power management control technology consists of a uniform flow control strategy and

\footnotetext{
+ Corresponding author. Tel.: 15502202810

E-mail address: 1626641299@qq.com
} 
a residual capacity matching control strategy to ensure safe and reliable power supply under different operating conditions, laying a foundation for the continuous and efficient work of the underwater robot.

\section{Hardware Design}

The hardware part of the operating system of underwater robot based on visible light communication technology is mainly composed of visible light communication module and underwater robot function module. The hardware design block diagram of the system is shown in Fig. 1:

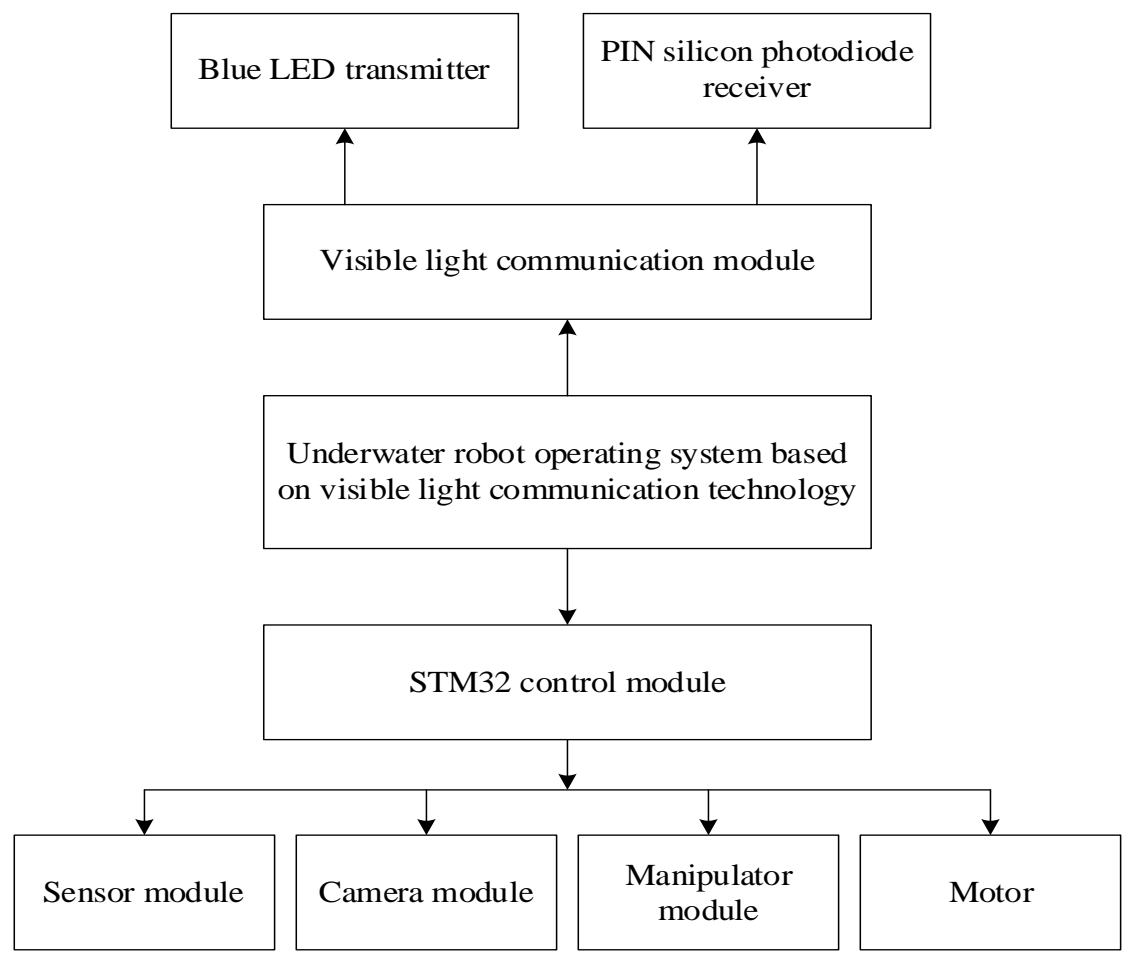

Fig. 1: The hardware design block diagram of the system.

\subsection{Visible Light Communication Module}

Visible light communication module is mainly composed of transmitting terminal and receiving end, since most of the water of the sea is blue, and the need of water molecules and dissolved salts and other substances to visible light absorption and scattering signal, in order to reduce visible in the process of transmission attenuation, and relevant scholars to explore the different wavelengths of light, studies have shown that the wavelength is $450-550 \mathrm{~nm}$ blue green light in the optical signal attenuation effect between the minimum[10-11], so the receiver illuminance is higher, so the blue LEDs as visible light communication module of the launch of the light source, the PIN silicon photoelectric diode as visible light signal at the receiving end. The LED lamp of this system directly emits high-speed modulated optical carrier signal invisible to human eyes through LED baseband modulation under the action of electric field by a semiconductor. The signal propagates freely in water, converts the optical signal into electrical signal through baseband demodulation, and communicates with other equipment and controls the operation of other equipment. The work flow chart is shown in Fig. 2:

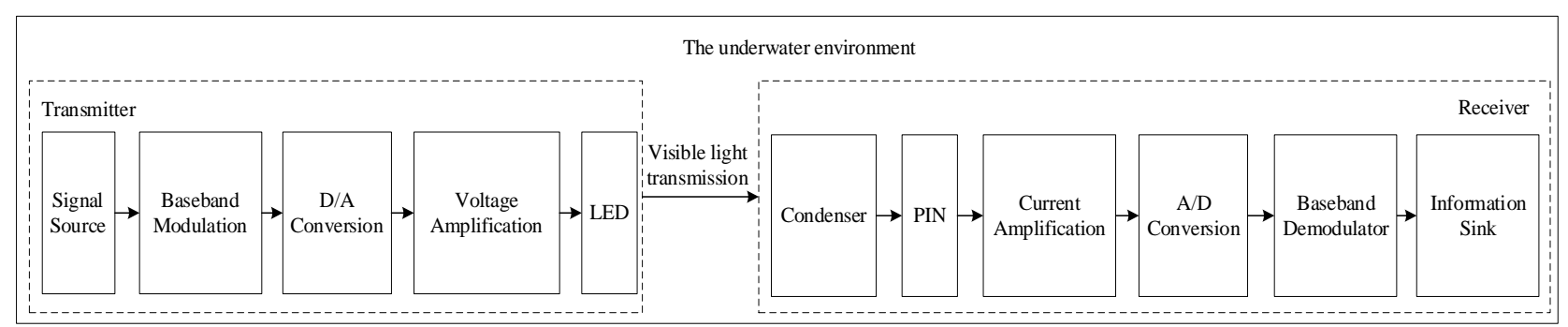

Fig. 2: The chart of visible light communication. 


\subsection{Underwater Robot Function Module}

The function module of underwater robot is mainly composed of STM32 single chip microcomputer control module, sensor module, camera module, manipulator module and motor. The STM32 single chip microcomputer control module adopts MPU6050 chip to control the attitude Angle of underwater robot.

\subsubsection{STM32}

This system uses STM32F103ZET6 MCU as the core controller to realize the underwater robot's attitude conversion, manipulator's grasping action and other functions, control the motor operation and the work of sensor module and camera module. The specific control process is shown in Fig. 3:

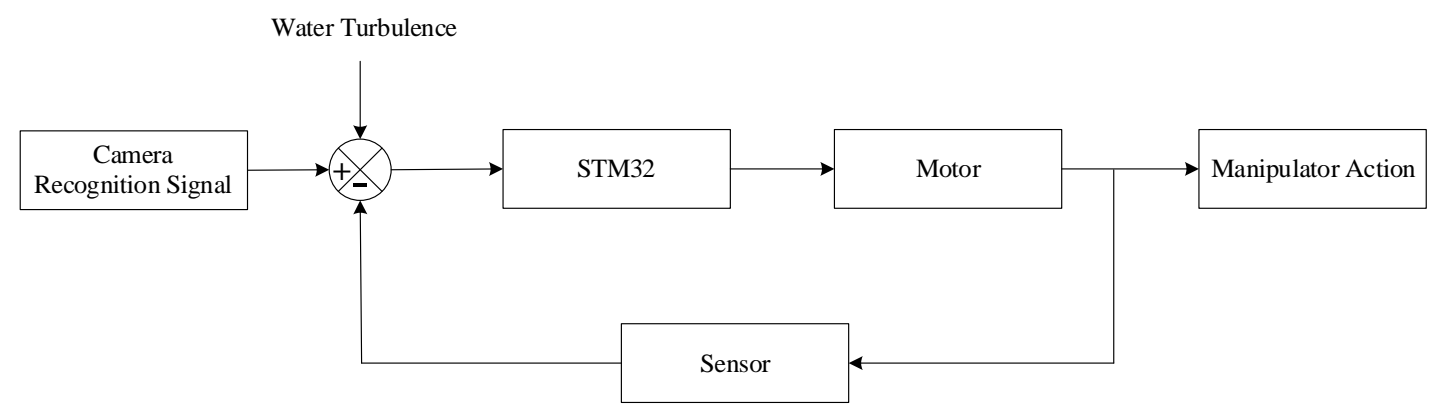

Fig. 3: The specific control process of STM32.

\subsubsection{MPU6050}

MPU6050 chip is a six-axis sensor module, which can simultaneously detect the motion data of triaxial acceleration, triaxial gyroscope (triaxial angular velocity) and temperature data, as shown in Fig. 4:

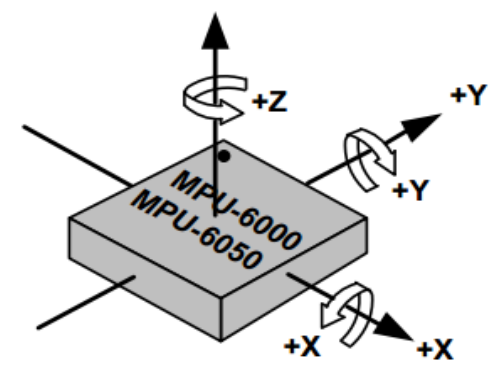

Fig. 4: Coordinates and direction of the MPU6050 sensor.

The DMP module inside the MPU6050 chip (Digital Motion Processor) can be used for filtering and fusion processing of sensor data. It directly outputs the attitude data after attitude solution to the master controller through $\mathrm{I}^{2} \mathrm{C}$ interface, thus reducing the calculation amount of the master controller. Its attitude resolution frequency can reach up to $200 \mathrm{~Hz}$, which is compatible for the field with high requirements for attitude control real-time. The schematic diagram of MPU6050 chip is shown in Fig. 5:

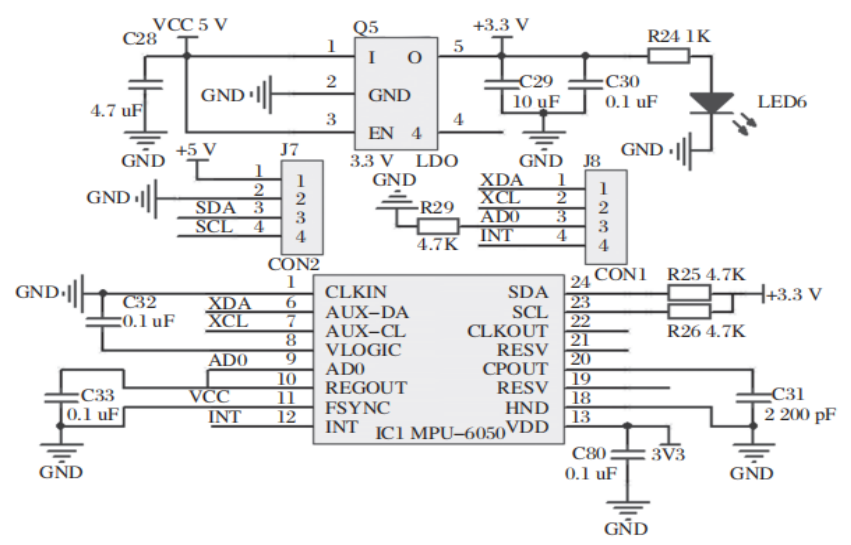

Fig. 5: The principle diagram of the MPU6050.

The attitude Angle is the Angle between the $\mathrm{X}, \mathrm{Y}$, and $\mathrm{Z}$ axes and the force vector $\mathrm{R}$. We define 
these angles as $A_{x r}, A_{y r}, A_{z r}, R_{x}, R_{y}$ and $R_{z}$ as the projection of $R$ vectors on the $X, Y$ and $Z$ axes. The spatial representation is shown in Fig. 6:

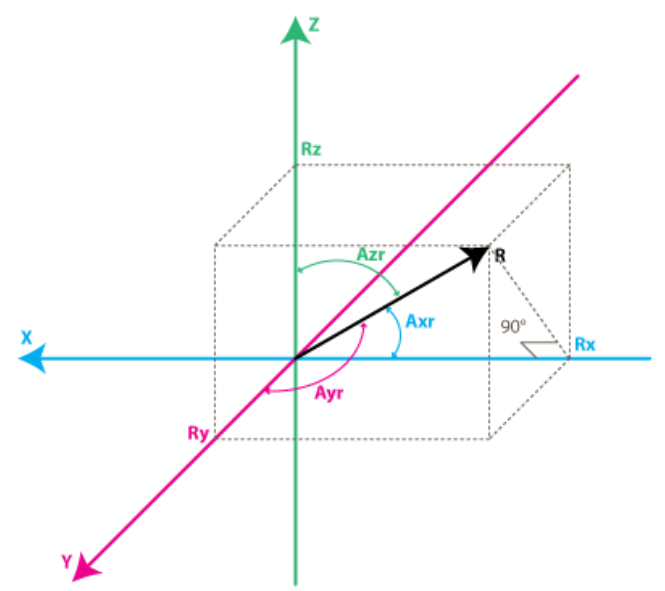

Fig. 6: Spatial representation of attitude Angle.

The relationship between $\mathrm{R}_{\mathrm{x}}, \mathrm{R}_{\mathrm{y}}$ and $\mathrm{R}_{\mathrm{z}}$ satisfies the formula:

$$
\mathrm{R}^{2}=\mathrm{R}_{\mathrm{x}}^{2}+\mathrm{R}_{\mathrm{y}}^{2}+\mathrm{R}_{\mathrm{z}}^{2}
$$

As shown in the figure above, the relationship between attitude angles of each axis and vector $\mathrm{R}$ is shown as follows:

$$
\begin{gathered}
\cos \left(\mathrm{A}_{\mathrm{xr}}\right)=\mathrm{R}_{\mathrm{x}} / \mathrm{R} \\
\cos \left(\mathrm{A}_{\mathrm{yr}}\right)=\mathrm{R}_{\mathrm{y}} / \mathrm{R} \\
\cos \left(\mathrm{A}_{\mathrm{zr}}\right)=\mathrm{R}_{\mathrm{z}} / \mathrm{R}
\end{gathered}
$$

The attitude Angle obtained after the reverse operation is:

$$
\begin{gathered}
\mathrm{A}_{\mathrm{xr}}=\arccos \left(\mathrm{R}_{\mathrm{x}} / \mathrm{R}\right) \\
\mathrm{A}_{\mathrm{yr}}=\arccos \left(\mathrm{R}_{\mathrm{y}} / \mathrm{R}\right) \\
\mathrm{A}_{\mathrm{zr}}=\arccos \left(\mathrm{R}_{\mathrm{z}} / \mathrm{R}\right)
\end{gathered}
$$

In the above formula, $R_{x}, R_{y}$ and $R_{z}$ are acceleration data of each axis respectively, and the calculation method is shown as follows:

Acceleration data $=$ original acceleration axis data / acceleration sensitivit $\mathrm{y}$

\section{Software Design}

The software part of the system uses Keil Uvision4 software to write programs and connect the software system with the hardware system. After the system is powered on, the system is divided into two parts. One part carries out visible light communication to realize wireless communication between the underwater robot and the upper computer, which is convenient for monitoring the operation of the underwater robot. The other part carries out the action execution of the underwater robot, and realizes the attitude conversion of the underwater robot and the execution of the corresponding action of the manipulator through the control of STM32 microcontroller. The specific working mode is shown in Fig. 7:

\section{Conclusions}

In this paper, the visible light communication technology as a means of communication, underwater robot and the upper machine to STM32 microcontroller as the core of underwater robot operating system controller, by controlling the motor, realization of underwater robot manipulator grasping motion sensors work, will MPU6050 chips used to control the position of underwater robot, can realize underwater robots currently three acceleration component and the calculation of the three rotating angular velocity. On the basis 
of realizing the attitude control of the underwater robot and the corresponding action of the manipulator, the system adopts the visible light communication technology to conduct wireless communication between the underwater robot and the upper computer, so as to realize the purpose of real-time detection of the seabed target and provide technical support for the limit operation of the underwater robot.

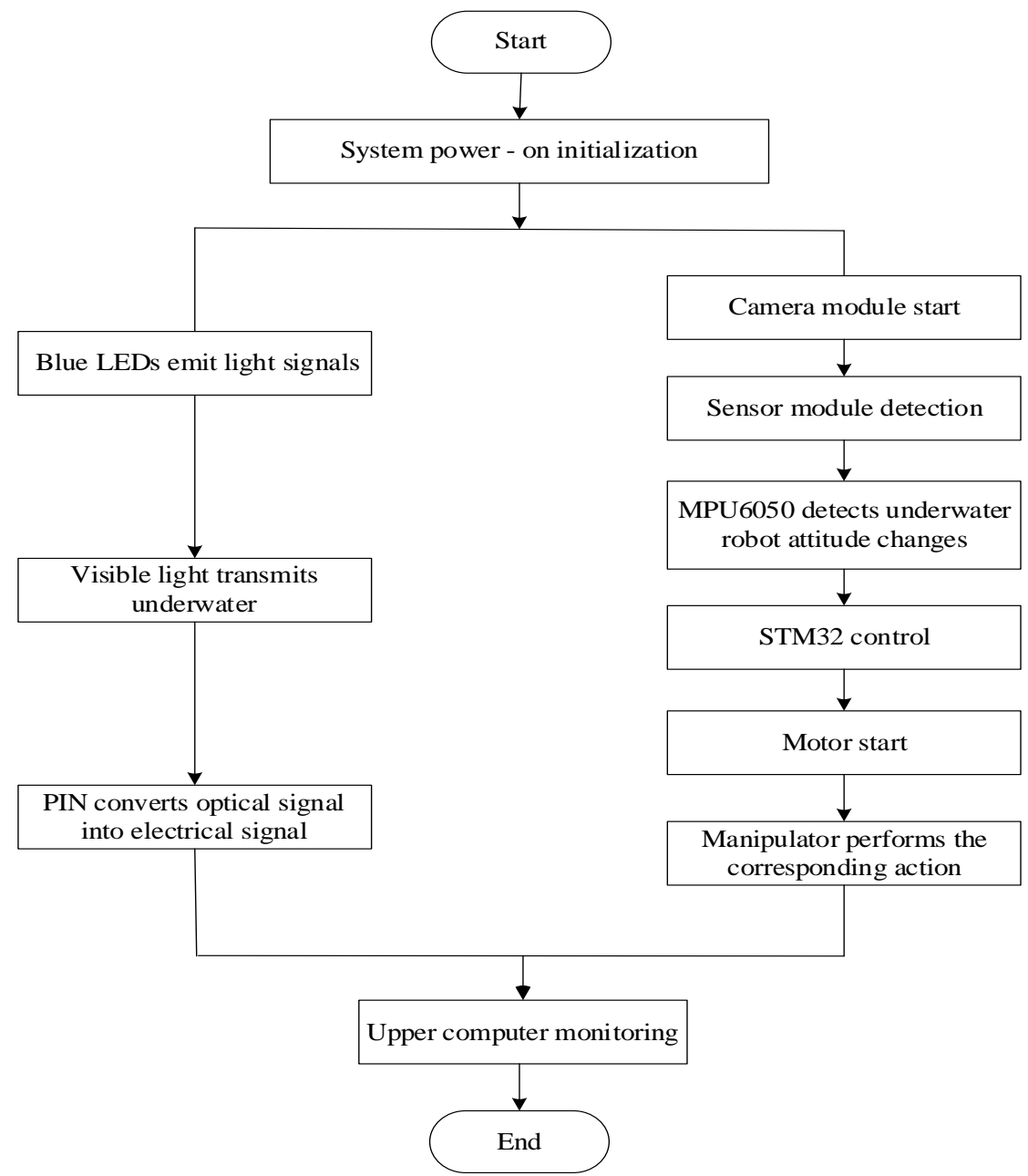

Fig. 7: The specific working mode.

\section{Acknowledgements}

This work is supported by National Key Research and Development Program of China (2017YFB0403603).

\section{References}

[1] Na Zhang, Qingqing Ren, Jianqiang He. Adaptive modulation channel estimation and coding for VLC system based on MIMO-OFDM. Optical Communication Technology, 2020, 1-7.

[2] Xiongbin Chen and Chengyu Min. Wireless communication that we can see-visible light communication. Physics, 2020, 49 (10): 688-696.

[3] Fangchen Hu and Nan Chi. The Principle, Key Technology and Applications of Underwater Visible Light Communication. China Lighting Electrical Appliances, 2018(01):6-13.

[4] Lin He, Huabiao Duan, Miao Chen, et al. Archives Room Management System Based on Visible Light Communication. Chinese Control and Decision Conference, 2020, 2323-2326.

[5] Yawei Wang. Design of an intelligent control System for underwater Cleaning Robot. Ship Materials and Market, 2020(09):23-24.

[6] Yuru Xu and Pengchao Li. Development trend of underwater robots. Nature, 2011, 33(03):125-132+2.

[7] Cheng Zhang. A Weapon to Conquer the unknown Seabed -- A Detailed Explanation of intelligent Underwater 
Robot. Robotics Industry, 2016(01):75-84.

[8] Gaochao Yan, Xiaoqin Shen, Fusheng Yu, et al. Optimization design of frame structure of ROV based on ANSYS-workbench. Manufacturing Automation, 2020, 42(10):1-3+7. 